\title{
NOTICIAS
}

\section{Construcción de una agenda política en defensa de la salud}

La transición hacia un nuevo milenio fue una ocasión excepcional para una profunda reflexión sobre los avances, desafíos y las posibles nuevas agendas para enfrentar los problemas identificados en materia de salud pública. Con esta perspectiva, la Asociación Latinoamericana de Medicina Social (Alames) y la International Association of Health Policy (IAHP) realizaron de manera conjunta el VIII Congreso Latinoamericano de Medicina Social y el XI Congreso de la Asociación Internacional de Políticas de Salud, respectivamente, alrededor del tema general: Globalización, reformas y equidad en salud.

Del 3 al 7 de julio de 2000, 657 delegados de 35 países se dieron cita en La Habana, Cuba, para avanzar en el análisis de los problemas contemporáneos de la medicina social y las políticas de salud, en la formulación de propuestas alternativas y de una agenda política que sirviera como ruta crítica en el futuro y como guía para la organización y movilización de grupos nacionales y regionales en defensa de la salud pública y del derecho ciudadano a la salud.

Se realizaron diversas actividades, a saber: la Conferencia "Juan César García", un curso precongreso,
15 talleres, el IV Encuentro de Egresados de la Maestría de Salud Pública de La Habana, cuatro páneles y seis mesas redondas; asimismo, se presentaron 13 libros, 527 comunicaciones coordinadas y 146 trabajos en cartel.

Las disertaciones en torno a la asociación que se establece entre globalización y salud explicitaron la existencia de un amplio fenómeno de mercantilización transfronteras de la salud en las Américas, expresión de un modelo hegemónico con fines de lucro que desarrolló el Norte hacia los países del Sur, y no de una conducta de solidaridad internacional capaz de superar las brechas de género y de promover el bienestar en las poblaciones más pobres del continente. En este contexto se discutió sobre aspectos tales como la actuación del aparato farmacéutico internacional en los países en desarrollo, las altas tasas de morbilidad y mortalidad perinatal de las mujeres maquiladoras, entre otros, que dan cuenta de las inequidades en salud y la globalización de los riesgos en el medio ambiente. Lo anterior, se señaló, constituye un dilema ético y moral, por lo que la salud global y la seguridad deben convertirse en la finalidad del desarrollo democrático de la humanidad.

En relación con las reformas en salud surgieron reflexiones en torno a la colusión entre los modelos de privatización en el sector salud, apoyados por las grandes organizaciones internacionales, y la necesidad de abrir nuevos mercados en el Sur ante la saturación de mercados seguros como el estadunidense; se destacó la relación entre las reformas y la salud reproductiva, y se caracterizaron las diferencias existentes entre los lenguajes de cada una de ellas: el primero, tecnocrático, mientras que el segundo se vincula al tema de derecho y ciudadanía, y tiene el reto de reducir las brechas en la provisión de servicios.

Acerca de la equidad en salud hubo consenso con relación al significado "acceso sin discriminación alguna a la atención en salud para aquellos que la necesiten". Otro concepto que se articula, con lo antes expuesto, es el de eficiencia, el que, de acuerdo con los panelistas, debe subordinarse a la meta de la equidad en salud. Los enfoques costo-beneficio actualmente utilizados han servido a las grandes organizaciones internacionales para justificar medidas como la privatización y la reducción del rol del Estado.

Los temas alrededor de los cuales giraron las comunicaciones coordinadas, las mesas redondas y los carteles, así como las propuestas o metas que surgieron de la discusión fueron: género y salud; descentralización y municipalización en salud; defensa de la vida, promoción de la salud y prevención de la enferme- 
dad; impacto de la globalización en el mundo del trabajo; formación de recursos humanos; economía y políticas de salud para el tercer milenio; condiciones de vida, ambiente y salud, y violencia como un problema de salud pública.

Entre los puntos que concretan la Agenda Política, producto de los trabajos desarrollados en los congresos mencionados, cabe señalar:

1. Constituir un observatorio para la evaluación de los procesos de reforma sanitaria que permita alimentar la construcción de alternativas para minimizar el impacto negativo en la salud.

2. Elaborar un informe sobre equidad y salud en América Latina que permita el debate público y la visibilidad de Alames como organización.

3. Organizar redes de trabajo permanente con los temas de globalización, reforma y equidad, permeadas por la perspectiva de género; consolidar las redes ya existentes y crear nuevas que permitan cumplir las metas de la agenda política.

4. Potenciar el papel de las universidades en la construcción de políticas de salud y evaluar el desempeño de los sistemas de salud a partir de la introducción de un paradigma social de la salud en sus currículos.

5. Promover alianzas, pactos y negociaciones entre las diferentes fuerzas sociales y políticas en función de la construcción social de la salud.

6. Desarrollar un sistema de información desagregada por sexo, que considere indicadores geográficos que den cuenta del impacto de la globalización en la salud y la calidad de vida.

7. Recuperar la noción eficiencia-eficacia en el logro de la equidad, accesibilidad y universalidad de la salud como derecho y expresión de ciudadanía.

Magda Luz Atrián Salazar. Edición, Salud Pública de México. 